\title{
Existence of Solutions for Fractional-Order Neutral Differential Inclusions with Impulsive and Nonlocal Conditions
}

\author{
Chao Song, ${ }^{1,2}$ Tao Zhu, ${ }^{1}$ and Jinde Cao ${ }^{2}$ \\ ${ }^{1}$ Department of Basic Courses, Nanjing Institute of Technology, Nanjing 211167, China \\ ${ }^{2}$ Research Center for Complex Systems and Network Sciences and Department of Mathematics, \\ Southeast University, Nanjing 210096, China \\ Correspondence should be addressed to Jinde Cao, jdcao@seu.edu.cn
}

Received 21 March 2012; Accepted 19 May 2012

Academic Editor: Zengji Du

Copyright (C) 2012 Chao Song et al. This is an open access article distributed under the Creative Commons Attribution License, which permits unrestricted use, distribution, and reproduction in any medium, provided the original work is properly cited.

This paper investigates the existence of solutions for fractional-order neutral impulsive differential inclusions with nonlocal conditions. Utilizing the fractional calculus and fixed point theorem for multivalued maps, new sufficient conditions are derived for ensuring the existence of solutions. The obtained results improve and generalize some existed results. Finally, an illustrative example is given to show the effectiveness of theoretical results.

\section{Introduction}

This paper deals with the existence of solutions for the following fractional-order differential inclusions with impulsive nonlocal conditions:

$$
\begin{gathered}
{ }^{c} D^{q}[x(t)-g(t, x(t))] \in F(t, x(t)), \quad t \in I=[0, T], t \neq t_{k}, \\
\left.\Delta x\right|_{t=t_{k}} \in I_{k}\left(x\left(t_{k}^{-}\right)\right), \quad k=1, \ldots, m, 0<t_{1}<\cdots<t_{m}<T, \\
x(0)=x_{0}+h(x),
\end{gathered}
$$

where $0<q<1$ and ${ }^{c} D^{q}$ is the Caputo fractional derivative. $I_{k}: R \rightarrow P(R)(k=1, \ldots, m$, and $F: I \times R \rightarrow D(R)$ are multivalued maps $(D(R)$ is the family of all nonempty subsets 
of $R) .\left.\Delta x\right|_{t=t_{k}}=x\left(t_{k}^{+}\right)-x\left(t_{k}^{-}\right), x\left(t_{k}^{+}\right), x\left(t_{k}^{-}\right)$represents the right and left limits of $x(t)$ at $t=t_{k}$, respectively. $g, h$ are given functions to be specified later.

The nonlocal problem was more general and has better effect than the classical Cauchy problems. So it has been studied extensively under various conditions in the literature [17] and the references contained therein. In [2], Byszewski and Lakshmikantham considered the existence and uniqueness of mild solutions when $f$ and $g$ satisfied Lipschitz conditions. In [3], Ntouyas and Tsamatos studied the case of compactness conditions of $g$ and $T(t)$. Liang et al. in [5] discussed the nonlinear nonlocal Cauchy problems when $T(t)$ was compact and $g$ was Lipschitz and $g$ was not Lipschitz and not compact, respectively. Xue in [6] established existence results of mild solutions for semilinear differential equations under various conditions on $f, g$, and $T(t)$. Subsequently in [7], the author examined the semilinear differential equations when $T(t)$ was compact and $g$ failed to be compact.

The theory of impulsive differential equations and differential inclusions has received much attention for the past decades because of its wide applicability in control, electrical engineering, mechanics, biology, and so on. For more details on this theory and applications, we refer to the monograph of Lakshmikantham et al. [8], Samoilenko and Perestyuk [9] and the references therein. In [10], Abada et al. discussed the impulsive differential inclusions with delays when $A$ was the infinitesimal generator of a strongly continuous semigroup $T(t)$. In the following work [11], they obtained some existence and controllability results when $A$ was a nondensely defined closed linear operator.

Since fractional-order differential equations have proved to be valuable tools in the modeling of many phenomena in physics and technical sciences, differential equations involving Riemann-Liouville as well as Caputo derivatives have been investigated extensively in the last decades (see [12-28] and the references therein). In [13], Mophou obtained the existence and uniqueness of mild solutions for the semilinear impulsive fractional differential equations by means of the Schauder fixed point theorem in Banach spaces. In [18], Benchohra and Slimani established sufficient conditions for the existence of solutions for a class of initial value problem for impulsive fractional differential equations. In [21], Chang and Nieto studied the existence of solutions for a class of fractional differential inclusions with boundary conditions by means of Bohnenblust-Karlin fixed point theorem. Balachandran et al. in [25] investigated fractional-order impulsive integrodifferential equations in Banach spaces. In [27], Agarwal and Ahmad studied the existence of solutions for nonlinear fractional differential equations and inclusions of order $q \in(3,4]$ with antiperiodic boundary conditions. The existence results were established for convex as well as nonconvex multivalued maps by some fixed point theorems, such as Leray-Schauder degree theory, and nonlinear alternative of Leray-Schauder type.

However, there is little information in the literature on neutral fractional-order impulsive differential inclusions with nonlocal conditions. Motivated by works mentioned above, we consider the existence results of (1.1) by using fixed point theorem for multivalued maps.

This paper is organized as follows. In Section 2, we will recall briefly some basic definitions and preliminary results which will be used throughout the paper. In Section 3, based on the fractional calculus and fixed point theorem for multivalued maps, we will prove the existence of solution to the problem (1.1). In Section 4, An example is given to show the effectiveness of our abstract results. 


\section{Preliminaries}

In this section, we introduce some definitions, notes, and preliminary facts which will be used in this paper.

Let $C(I, R)$ be a Banach space, with the norm $\|x\|_{C}=\sup \{|x(t)|, t \in I\}$. Let $L^{p}(I, R)$ denote the space of Bochner integrable functions on $I$ with the norm $\|x\|_{L^{p}}=\left(\int_{0}^{T}|x(t)|^{p} d t\right)^{1 / p}$.

Let $(X,\|\cdot\|)$ be a Banach space. $P(X)$ is the family of all nonempty subsets of $X$. $P_{\mathrm{bd}}(\mathrm{X}), P_{\mathrm{cl}}(\mathrm{X}), P_{\mathrm{bd}, \mathrm{cl}}(\mathrm{X}), P_{\mathrm{cp}, \mathrm{cv}}(\mathrm{X})$, and $P_{\mathrm{bd}, \mathrm{cl}, \mathrm{cv}}(\mathrm{X})$ denote, respectively, the family of all nonempty bounded, closed, bounded-closed, compact-convex, and bounded-closed-convex subsets of $X$. A multivalued map $G: X \rightarrow P(X)$ is convex (closed) valued if $G(x)$ is convex (closed) for all $x \in X$. We say that $G$ is bounded on bounded sets if $G(B)=\cup_{x \in B} G(x)$ is bounded in $X$ for all $B \in P_{\mathrm{bd}}(X)$.

A mapping $G$ is called upper semicontinuous (u.s.c.) on $X$ if, for each $x_{0} \in X$, the set $G\left(x_{0}\right)$ is a nonempty closed subset of $X$ and if, for each open set $N$ of $X$ containing $G\left(x_{0}\right)$, there exists an open neighborhood $N_{0}$ of $x_{0}$ such that $G\left(N_{0}\right) \subseteq N$. G is completely continuous if $G(V)$ is relatively compact for every $V \in P_{\mathrm{bd}}(X)$. If the multivalued map $G$ is completely continuous with nonempty compact values, then $G$ is u.s.c. if and only if $G$ has a closed graph (i.e., $x_{n} \rightarrow x_{*}, y_{n} \rightarrow y_{*}, y_{n} \in G\left(x_{n}\right)$ imply $y_{*} \in G\left(x_{*}\right)$ ).

A mapping $G$ has a fixed point if there exists $x \in X$ such that $x \in G(x)$. A multivalued map $G: I \rightarrow P_{\mathrm{cl}}(X)$ is said to be measurable if, for each $x \in X$, the function

$$
t \longmapsto d(x, G(t))=\inf \{|x-z|: z \in G(t)\}
$$

is measurable.

Definition 2.1. A multivalued map $F: I \times X \rightarrow P(X)$ is $L^{1}$-Carathéodory if

(i) $t \mapsto F(t, u)$ is measurable for each $u \in X$;

(ii) $u \mapsto F(t, u)$ is upper semicontinuous for almost all $t \in I$;

(iii) for each $q>0$, there exists $\phi_{q} \in L^{1}\left(I, R^{+}\right)$such that

$$
\|F(t, u)\|_{p}=\sup \{\|v\|: v \in F(t, u)\} \leq \phi_{q}(t)
$$

for all $\|u\| \leq q$ and a.e. $t \in I$.

Definition 2.2. A multivalued operator $N: X \rightarrow P_{\mathrm{cl}}(X)$ is called

(a) $\gamma$-Lipschitz if there exists $\gamma>0$ such that

$$
H_{d}(N(x), N(y)) \leq r d(x, y), \quad x, y \in X ;
$$

(b) a contraction if it is $\gamma$-Lipschitz with $\gamma<1$.

The key tool in our approach is the following fixed point theorem. 
Lemma 2.3 (see [29]). Let $B(0, r)$ and $B[0, r]$ denote, respectively, the open and closed balls in $a$ Banach space $X$ centered at origin and of radius $r$, and let $\Phi_{1}: X \rightarrow P_{\mathrm{bd}, \mathrm{cl}, \mathrm{cv}}(X)$ and $\Phi_{2}: B[0, r] \rightarrow$ $P_{c p, c v}(X)$ be two multivalued operators satisfying the following:

(a) $\Phi_{1}$ is a contraction,

(b) $\Phi_{2}$ is completely continuous.

Then either

(i) the operator inclusion $x \in \Phi_{1} x+\Phi_{2} x$ has a solution in $B[0, r]$ or

(ii) there exists an $u \in X$ with $\|u\|=r$ such that $\lambda u \in \Phi_{1} u+\Phi_{2} u$, for some $\lambda>1$.

Lemma 2.4 (see [30]). Let I be a compact real interval and $X$ a Banach space. Let $F$ be an $L^{1-}$ Caratheodory multivalued mapping with compact convex values with $S_{F, y} \neq \emptyset$, where $S_{F, y}=\{f \in$ $L^{1}(I, X): f(t) \in F(t, y(t))$, a.e.t $\left.\in I\right\}$ and let $\Gamma: L^{1}(I, X) \rightarrow C(I, X)$ be a linear continuous mapping. Then the operator

$$
\Gamma \circ S_{F}: C(I, X) \longrightarrow P_{\mathrm{cp}, \mathrm{cv}}(C(I, X)), \quad x \longmapsto\left(\Gamma \circ S_{F}\right)(x)=\Gamma\left(S_{F, x}\right)
$$

is a closed graph operator in $C(I, X) \times C(I, X)$.

For more details on multivalued map, see the books of Aubin and Cellina [31], Deimling [32].

Now, we recall some definitions and facts about fractional derivatives and fractional integrals of arbitrary order, see [33].

Definition 2.5. The Riemann-Liouville fractional integral operator of order $q>0$ of a function $h$ is defined by

$$
I_{a}^{q} h(t)=\frac{1}{\Gamma(q)} \int_{a}^{t}(t-s)^{q-1} h(s) d s
$$

where $\Gamma$ is the gamma function.

Definition 2.6. The Caputo fractional-order derivative of order $q>0$ of a function $h(t)$ is defined by

$$
\left({ }^{c} D_{a+}^{q} h\right)(t)=\frac{1}{\Gamma(n-q)} \int_{a}^{t}(t-s)^{n-q-1} h^{(n)}(s) d s, \quad n-1<q<n, n \in \mathbb{N}^{+} .
$$

Lemma 2.7 (see [34]). Let $q>0$; then the differential equation

$$
{ }^{c} D^{q} y(t)=h(t)
$$

has solutions $y(t)=I^{q} h(t)+c_{0}+c_{1} t+c_{2} t^{2}+\cdots+c_{n-1} t^{n-1}$, where $c_{i} \in R, n=[q]+1$. 
Lemma 2.8 ( see [34]). Let $\alpha>0$; then

$$
I^{\alpha c} D^{\alpha} h(t)=h(t)+a_{0}+a_{1} t+a_{2} t^{2}+\cdots+a_{n-1} t^{n-1}
$$

for some $a_{i} \in R, i=0,1,2, \ldots, n-1, n=[\alpha]+1$.

\section{Main results}

In this section, main results are presented.

Let $P C(I, R)=\left\{x: I \rightarrow R: x \in C\left(\left(t_{k-1}, t_{k}\right], R\right), k=0,1, \ldots, m\right.$, and there exist $x\left(t_{k}^{-}\right), x\left(t_{k}^{+}\right), k=1, \ldots, m$ with $\left.x\left(t_{k}^{-}\right)=x\left(t_{k}\right)\right\}$. This set is a Banach space with the norm $\|x\|_{P C}=\sup _{t \in I}|x(t)|$.

As a consequence of Lemmas 2.7 and 2.8, we can define the solution of problem (1.1).

Definition 3.1. The function $x \in P C(I, R)$ is said to be a solution of the problem (1.1) if $x(0)=$ $x_{0}+h(x)$, and there exist $f(t) \in S_{F, x}, J_{k} \in I_{k}\left(x\left(t_{k}^{-}\right)\right)$such that integral equation

$$
\begin{aligned}
x(t)= & x_{0}+h(x)-g(0, x(0))+g(t, x(t))+\frac{1}{\Gamma(q)} \sum_{0<t_{i}<t} \int_{t_{i-1}}^{t_{i}}\left(t_{i}-s\right)^{q-1} f(s) d s \\
& +\frac{1}{\Gamma(q)} \int_{t_{i}}^{t}(t-s)^{q-1} f(s) d s+\sum_{0<t_{i}<t} J_{k}, \quad t \in I,
\end{aligned}
$$

is satisfied.

For the study of the system (1.1), the following hypotheses are given:

(H1) $F$ is a Carathéodory map with compact convex values, and for each fixed $x \in$ $R, S_{F, x}$ is nonempty;

(H2) there exist a function $a(\cdot) \in L^{p}\left(I, R^{+}\right)$, where $p>1 / q, p \in \mathbb{N}^{+}$and a nondecreasing continuous function $\Omega: R^{+} \rightarrow R^{+}$such that

$$
\|F(t, x)\|_{p}=\sup \{|f(t)|: f(t) \in F(t, x(t))\} \leq a(t) \Omega(\|x\|),
$$

for $t \in I$ and each $x \in R$;

(H3) $g: I \times R \rightarrow R$ is Lipschtiz continuous with Lipschtiz constant $l_{g}$, and there exists $M>0$ such that $|g(t, x(t))| \leq M$;

$(\mathrm{H} 4) h: P C(I, R) \rightarrow R$ is Lipschtiz continuous with Lipschtiz constant $l_{h}$;

(H5) $I_{k}: R \rightarrow P(R)(k=1, \ldots, m)$ are multivalued maps with bounded, closed, and convex values. And there exist $c_{k}>0, k=1, \ldots, m$, such that

$$
H_{d}\left(I_{k}(y), I_{k}(x)\right) \leq c_{k}\|y-x\|
$$

for all $x, y \in R$; 
(H6) $C=((p-1) /(p q-1))^{(p-1) / p} T^{q-1 / p}, C_{0}=\left|x_{0}\right|+|h(0)|+2 M+\sum_{k=1}^{m}\left|I_{k}(0)\right|$,

$$
l_{h}+2 l_{g}+\sum_{k=1}^{m} c_{k}<1
$$

The existence of solutions is now presented.

Theorem 3.2. Assume that (H1)-(H6) are satisfied; then the problem (1.1) has at least one solution on I provided that there exists a real number $r>0$ such that

$$
\frac{C_{0} \Gamma(q)+C(m+1) \Omega(r)\|a\|_{L^{p}}}{\left(1-l_{h}-\sum_{k=1}^{m} c_{k}\right) \Gamma(q)}<r
$$

Proof. We transform the problem (1.1) into a fixed point problem by considering the multivalued operator $N(x): P C(I, R) \rightarrow P(P C(I, R))$ :

$$
\begin{gathered}
N(x)=\left\{\varphi \in P C(I, R): \varphi(t)=x_{0}+h(x)-g(0, x(0))+g(t, x(t))+\sum_{0<t_{i}<t} J_{k}\right. \\
\left.+\frac{1}{\Gamma(q)} \sum_{0<t_{i}<t} \int_{t_{i-1}}^{t_{i}}\left(t_{i}-s\right)^{q-1} f(s) d s+\frac{1}{\Gamma(q)} \int_{t_{i}}^{t}(t-s)^{q-1} f(s) d s\right\},
\end{gathered}
$$

where $f \in S_{F, x}, J_{k} \in I_{k}\left(x\left(t_{k}^{-}\right)\right), k=1, \ldots, m$.

Consider the multivalued operators $\alpha, \beta$ :

$$
\begin{gathered}
\alpha(x)=\left\{\varphi \in P C(I, R): \varphi(t)=x_{0}+h(x)-g(0, x(0))+g(t, x(t))+\sum_{0<t_{i}<t} J_{k}\right\}, \\
\beta(x)=\left\{\varphi \in P C(I, R): \varphi(t)=\frac{1}{\Gamma(q)} \sum_{0<t_{i}<t} \int_{t_{i-1}}^{t_{i}}\left(t_{i}-s\right)^{q-1} f(s) d s+\frac{1}{\Gamma(q)} \int_{t_{i}}^{t}(t-s)^{q-1} f(s) d s\right\} .
\end{gathered}
$$

It is clear that $N=\alpha+\beta$. This problem of finding a solution of (1.1) is reduced to find a solution of the operator inclusions $x \in \alpha(x)+\beta(x)$. In what follows, we aim to show that the operator $\alpha+\beta$ has a fixed point, which is a solution of the system (1.1). For this purpose, We will show that $\alpha+\beta$ satisfies all the conditions of Lemma 2.3. For better readability, the proof will be given in several steps. is not true.

Step 1. A priori bounded on solution: we show that the second assertion of Lemma 2.3 
Define an open ball $B(0, r) \in P C(I, R)$, where the real number $r$ satisfies the inequality given in condition (3.5). Let $x$ be a possible solution of $\lambda x \in \alpha(x)+\beta(x)$, for some real number $\iota>1$ with $\|x\|=r$. Then we have

$$
\begin{aligned}
x(t) \in \lambda^{-1} & \left(x_{0}+h(x)-g(0, x(0))+g(t, x(t))+\sum_{0<t_{i}<t} J_{k}\right) \\
& +\frac{\lambda^{-1}}{\Gamma(q)} \sum_{0<t_{i}<t} \int_{t_{i-1}}^{t_{i}}\left(t_{i}-s\right)^{q-1} f(s) d s+\frac{\lambda^{-1}}{\Gamma(q)} \int_{t_{i}}^{t}(t-s)^{q-1} f(s) d s .
\end{aligned}
$$

Hence by $(\mathrm{H} 2)-(\mathrm{H} 5)$, we have

$$
\begin{aligned}
|x(t)| \leq & \left|x_{0}+h(x)-g(0, x(0))+g(t, x(t))+\sum_{0<t_{i}<t} J_{k}\right| \\
& +\frac{1}{\Gamma(q)} \sum_{0<t_{i}<t} \int_{t_{i-1}}^{t_{i}}\left(t_{i}-s\right)^{q-1}|f(s)| d s+\frac{1}{\Gamma(q)} \int_{t_{i}}^{t}(t-s)^{q-1}|f(s)| d s \\
\leq & \left|x_{0}\right|+|h(0)|+l_{h}\|x\|+2 M+\sum_{k=1}^{m} c_{k}\left|x\left(t_{k}^{-}\right)\right|+\sum_{k=1}^{m}\left|I_{k}(0)\right| \\
& +\frac{1}{\Gamma(q)} \sum_{0<t_{i}<t} \int_{t_{i-1}}^{t_{i}}\left(t_{i}-s\right)^{q-1} a(s) \Omega(\|x\|) d s+\frac{1}{\Gamma(q)} \int_{t_{i}}^{t}(t-s)^{q-1} a(s) \Omega(\|x\|) d s \\
\leq & C_{0}+\left(l_{h}+\sum_{k=1}^{m} c_{k}\right)\|x\|+\frac{C(m+1) \Omega(\|x\|)\|a\|_{L^{p}}}{\Gamma(q)} .
\end{aligned}
$$

Taking the supremum over $t$, we get

$$
\|x\| \leq C_{0}+\left(l_{h}+\sum_{k=1}^{m} c_{k}\right)\|x\|+\frac{C(m+1) \Omega(\|x\|)\|a\|_{L^{p}}}{\Gamma(q)} .
$$

Substituting $\|x\|=r$ in the above inequality yields

$$
r \leq \frac{C_{0} \Gamma(q)+C(m+1) \Omega(\|r\|)\|a\|_{L^{p}}}{\left(1-l_{h}-\sum_{k=1}^{m} c_{k}\right) \Gamma(q)}
$$

which is a contradiction to (3.5). 
Step 2. $\alpha: P C(I, R) \rightarrow P(P C(I, R))$ is a contraction mapping.

Indeed, let $x_{1}, x_{2} \in P C(I, R)$, and from (H5), we have

$$
\begin{aligned}
& H_{d}\left(\alpha\left(x_{1}\right), \alpha\left(x_{2}\right)\right)= H_{d}\left(x_{0}+h\left(x_{1}\right)-g\left(0, x_{1}(0)\right)+g\left(t, x_{1}(t)\right)+\sum_{0<t_{i}<t} I_{k}\left(x_{1}\left(t_{k}^{-}\right)\right)\right. \\
&\left.x_{0}+h\left(x_{2}\right)-g\left(0, x_{2}(0)\right)+g\left(t, x_{2}(t)\right)+\sum_{0<t_{i}<t} I_{k}\left(x_{2}\left(t_{k}^{-}\right)\right)\right) \\
& \leq H_{d}\left(h\left(x_{1}\right), h\left(x_{2}\right)\right)+H_{d}\left(-g\left(0, x_{1}(0)\right),-g\left(0, x_{2}(0)\right)\right) \\
&+H_{d}\left(g\left(t, x_{1}(t)\right), g\left(t, x_{2}(t)\right)\right)+H_{d}\left(\sum_{0<t_{i}<t} I_{k}\left(x_{1}\left(t_{k}^{-}\right)\right), \sum_{0<t_{i}<t} I_{k}\left(x_{2}\left(t_{k}^{-}\right)\right)\right) \\
& \leq\left(l_{h}+2 l_{g}+\sum_{k=1}^{m} c_{k}\right)\left\|x_{1}-x_{2}\right\| .
\end{aligned}
$$

Hence by (3.4), $\alpha$ is a contraction mapping.

Step 3. $\beta$ has compact, convex values, and it is completely continuous.

Claim 1. $\beta$ is convex for each $x \in P C(I, R)$.

In fact, if $\varphi_{1}$ and $\varphi_{2}$ belong to $\beta(x)$, then there exist $f_{1}, f_{2} \in S_{F, x}$ such that

$$
\varphi_{i}(t)=\frac{1}{\Gamma(q)} \sum_{0<t_{i}<t} \int_{t_{i-1}}^{t_{i}}\left(t_{i}-s\right)^{q-1} f_{i}(s) d s+\frac{1}{\Gamma(q)} \int_{t_{i}}^{t}(t-s)^{q-1} f_{i}(s) d s, \quad i=1,2 .
$$

Let $0 \leq \lambda \leq 1$. Then, for each $t \in I$, we have

$$
\begin{aligned}
\left(\lambda \varphi_{1}+(1-\lambda) \varphi_{2}\right)(t)= & \frac{1}{\Gamma(q)} \sum_{0<t_{i}<t} \int_{t_{i-1}}^{t_{i}}\left(t_{i}-s\right)^{q-1}\left(\lambda f_{1}(s)+(1-\lambda) f_{2}(s)\right) d s \\
& +\frac{1}{\Gamma(q)} \int_{t_{i}}^{t}(t-s)^{q-1}\left(\lambda f_{1}(s)+(1-\lambda) f_{2}(s)\right) d s .
\end{aligned}
$$

Since $S_{F, x}$ is convex (because $F$ has convex values), we have

$$
\lambda \varphi_{1}+(1-\lambda) \varphi_{2} \in \beta(x)
$$

Claim 2. $\beta$ maps bounded sets into bounded sets in $P C(I, R)$. Let $r>0, B_{r}=\{x \in P C(I, R)$ : $\|x\| \leq r\}$ be a bounded set in $P C(I, R)$. For $x \in B_{r}, \varphi \in \beta(x)$, then there exists $f \in S_{F, x}$ such that

$$
\varphi(t)=\frac{1}{\Gamma(q)} \sum_{0<t_{i}<t} \int_{t_{i-1}}^{t_{i}}\left(t_{i}-s\right)^{q-1} f(s) d s+\frac{1}{\Gamma(q)} \int_{t_{i}}^{t}(t-s)^{q-1} f(s) d s
$$


Therefore,

$$
\begin{aligned}
|\varphi(t)| & \leq \frac{1}{\Gamma(q)} \sum_{0<t_{i}<t} \int_{t_{i-1}}^{t_{i}}\left(t_{i}-s\right)^{q-1}|f(s)| d s+\frac{1}{\Gamma(q)} \int_{t_{i}}^{t}(t-s)^{q-1}|f(s)| d s \\
& \leq \frac{1}{\Gamma(q)} \sum_{k=1}^{m} \int_{t_{i-1}}^{t_{i}}\left(t_{i}-s\right)^{q-1} a(s) \Omega(\|x\|) d s+\frac{1}{\Gamma(q)} \int_{t_{i}}^{t}(t-s)^{q-1} a(s) \Omega(\|x\|) d s \\
& \leq \frac{C(m+1) \Omega(r)\|a\|_{L^{p}}}{\Gamma(q)} .
\end{aligned}
$$

Thus

$$
\|\varphi\| \leq \frac{C(m+1) \Omega(r)\|a\|_{L^{p}}}{\Gamma(q)}:=m^{*}
$$

Claim 3. $\beta$ maps bounded sets into equicontinuous sets. Let $\tau_{1}, \tau_{2} \in I, \tau_{1}<\tau_{2}, B_{r}$ be a bounded set of $P C(I, R)$ as in Claim 2 and $x \in B_{r}$. Then there exists $f \in S_{F, x}$ such that

$$
\varphi(t)=\frac{1}{\Gamma(q)} \sum_{0<t_{i}<t} \int_{t_{i-1}}^{t_{i}}\left(t_{i}-s\right)^{q-1} f(s) d s+\frac{1}{\Gamma(q)} \int_{t_{i}}^{t}(t-s)^{q-1} f(s) d s .
$$

By elementary computation, we have

$$
\begin{aligned}
\left|\varphi\left(\tau_{2}\right)-\varphi\left(\tau_{1}\right)\right| \leq & \frac{1}{\Gamma(q)} \int_{t_{i}}^{\tau_{1}}\left|\left(\tau_{2}-s\right)^{q-1}-\left(\tau_{1}-s\right)^{q-1}\|f(s)\| d s+\frac{1}{\Gamma(q)} \int_{\tau_{1}}^{\tau_{2}}\left(\tau_{2}-s\right)^{q-1}\right| f(s) \mid d s \\
\leq & \frac{\|a\|_{L^{p}} \Omega(r)}{\Gamma(q)}\left(\int_{t_{i}}^{\tau_{1}}\left[\left(\tau_{2}-s\right)^{q-1}-\left(\tau_{1}-s\right)^{q-1}\right]^{p /(p-1)} d s\right)^{(p-1) / p} \\
& +\frac{\|a\|_{L^{p}} \Omega(r)}{\Gamma(q)}\left(\int_{\tau_{1}}^{\tau_{2}}\left(\tau_{2}-s\right)^{(p q-p) /(p-1)} d s\right)^{(p-1) / p} \\
\leq & \frac{\|a\|_{L^{p}} \Omega(r)}{\Gamma(q)}\left(\frac{p-1}{p q-1}\right)^{(p-1) / p} \\
& \times\left[\left(\tau_{2}-\tau_{1}\right)^{(p q-1) /(p-1)}-\left(\tau_{2}-t_{i}\right)^{(p q-1) /(p-1)}+\left(\tau_{1}-t_{i}\right)^{(p q-1) /(p-1)}\right]^{(p-1) / p} \\
& +\frac{\|a\|_{L^{p}} \Omega(r)}{\Gamma(q)}\left(\frac{p-1}{p q-1}\right)^{(p-1) / p}\left(\tau_{2}-\tau_{1}\right)^{(p q-1) / p} .
\end{aligned}
$$

As $\tau_{1} \rightarrow \tau_{2}$, the right-hand side of the above inequality tends to zero. As a consequence of Claims 1 to 3 together with the Arzelá-Ascoli theorem, we conclude that $\beta$ is completely continuous. 
Claim 4. $\beta$ has a closed graph. Let $x_{n} \rightarrow x_{*}, \varphi_{n} \in \beta\left(x_{n}\right)$, and $\varphi_{n} \rightarrow \varphi_{*} ;$ we prove that $\varphi_{*} \in$ $\beta\left(x_{*}\right)$. Now $\varphi_{n} \in \beta\left(x_{n}\right)$ means that there exists $f_{n} \in S_{F, x_{n}}$ such that

$$
\varphi_{n}(t)=\frac{1}{\Gamma(q)} \sum_{0<t_{i}<t} \int_{t_{i-1}}^{t_{i}}\left(t_{i}-s\right)^{q-1} f_{n}(s) d s+\frac{1}{\Gamma(q)} \int_{t_{i}}^{t}(t-s)^{q-1} f_{n}(s) d s, \quad t \in I .
$$

We will prove that there exists $f_{*} \in S_{F, x_{*}}$ such that

$$
\varphi_{*}(t)=\frac{1}{\Gamma(q)} \sum_{0<t_{i}<t} \int_{t_{i-1}}^{t_{i}}\left(t_{i}-s\right)^{q-1} f_{*}(s) d s+\frac{1}{\Gamma(q)} \int_{t_{i}}^{t}(t-s)^{q-1} f_{*}(s) d s .
$$

Consider the continuous operator $\kappa: L^{1}(I, X) \rightarrow C(I, X)$

$$
(\kappa f)(t)=\frac{1}{\Gamma(q)} \sum_{0<t_{i}<t} \int_{t_{i-1}}^{t_{i}}\left(t_{i}-s\right)^{q-1} f(s) d s+\frac{1}{\Gamma(q)} \int_{t_{i}}^{t}(t-s)^{q-1} f(s) d s .
$$

From Lemma 2.4, it follows that $\kappa \circ S_{F}$ is a closed graph operator. Since $\varphi_{n} \in \mathcal{\kappa} \circ S_{F, x_{n}}$, we have $\varphi_{*} \in \kappa \circ S_{F, x_{*}}$. That is, there exists $f_{*} \in S_{F, x_{*}}$ such that

$$
\varphi_{*}(t)=\frac{1}{\Gamma(q)} \sum_{0<t_{i}<t} \int_{t_{i-1}}^{t_{i}}\left(t_{i}-s\right)^{q-1} f_{*}(s) d s+\frac{1}{\Gamma(q)} \int_{t_{i}}^{t}(t-s)^{q-1} f_{*}(s) d s
$$

Therefore, $\beta$ has a closed graph.

As a consequence of Lemma 2.3, the operator inclusion $x \in \alpha(x)+\beta(x)$ has a solution which implies that the problem (1.1) has a solution on $I$.

Remark 3.3. If we take $F(t, x)=\{f(t, x)\}$, where $f$ is a continuous operator, then the result corresponds to a single-value problem. In this case, our result is also new in the present configuration.

Corollary 3.4. Assume that (H1)-(H6) are satisfied; then the problem (1.1) has at least one solution on I provided that

$$
\|a\|_{L^{p}}<\liminf _{R \rightarrow+\infty} \frac{R\left(1-l_{h}-\sum_{k=1}^{m} c_{k}\right)-C_{0} \Gamma(q)}{C(m+1) \Omega(R)} .
$$

Proof. From (3.25), we know that there exists a constant $r>0$, such that

$$
\|a\|_{L^{p}}<\frac{r\left(1-l_{h}-\sum_{k=1}^{m} c_{k}\right)-C_{0} \Gamma(q)}{C(m+1) \Omega(r)} .
$$

Since the proof of Corollary 3.4 follows the very same lines as the proof of Theorem 3.2, we do not give details. 
Corollary 3.5. Assume that (H1), (H3)-(H6), and the following condition are satisfied,

$\left(H 2^{\prime}\right)$ There exists a function $\rho(\cdot) \in L^{p}(I, R)$, such that

$$
\|F(t, x)\|_{p}=\sup \{|f(t)|: f(t) \in F(t, x(t))\} \leq \rho(t)\|x\|,
$$

for $t \in I$ and each $x \in R$.

Then the problem (1.1) has at least one solution on I provided that

$$
\|\rho\|_{L^{p}}<\frac{1-l_{h}-\sum_{k=1}^{m} c_{k}}{C(m+1)}
$$

Proof. In this case, we take $a(t)=\rho(t), \Omega(x)=x$ in Corollary 3.4.

At last we would like to discuss the impulsive condition.

$\left(\mathrm{H}^{\prime}\right) I_{k}: R \rightarrow R(k=1, \ldots, m)$ are single continuous operator, and there exists $c_{k}>$ $0, k=1, \ldots, m$, such that $\left|I_{k}(y)-I_{k}(x)\right| \leq c_{k}|y-x|$, for all $x, y \in R$.

Corollary 3.6. Assume that (H1)-(H4), (H5'), (H6) are satisfied; then the problem (1.1) has at least one solution on I provided that

$$
\|a\|_{L^{p}}<\liminf _{R \rightarrow+\infty} \frac{R\left(1-l_{h}-\sum_{k=1}^{m} c_{k}\right)-C_{0} \Gamma(q)}{C(m+1) \Omega(R)} .
$$

\section{An Illustrative Example}

In this section, an example is given to show the effectiveness of our theoretical result. Consider the following fractional-order differential inclusions with impulsive and nonlocal conditions:

$$
\begin{gathered}
{ }^{c} D^{q}\left[x(t)-\frac{e^{-t}|x(t)|}{\left(9+e^{t}\right)(1+|x(t)|)}\right] \in F(t, x(t)), \quad t \in I=[0,1], t \neq \frac{1}{2}, \\
\left.\Delta x\right|_{t=1 / 2}=\frac{\left|x\left(1 / 2^{-}\right)\right|}{3+\left|x\left(1 / 2^{-}\right)\right|} \\
x(0)=h(x),
\end{gathered}
$$

where $h(x)=(1 / 9) x(0)+(1 / 10) x(1), g(t, x)=\left(e^{-t} x\right) /\left(9+e^{t}\right)(1+x), I_{k}(x)=x /(3+x), x \in$ $[0, \infty)$, and $F: I \times R \rightarrow P(R)$ is a multivalued map given by

$$
x \rightarrow F(t, x)=\left[\frac{e^{-2 t}|x(t)|}{\left(9+e^{t}\right)(1+|x(t)|)}, \frac{e^{-t}|x(t)|}{\left(9+e^{t}\right)(1+|x(t)|)}\right] .
$$


Thus,

$$
\|F(t, x)\|_{p}=\sup \{|f(t)|: f(t) \in F(t, x(t))\} \leq a(t) \Omega(\|x\|)
$$

where $a(t)=1 /\left(9+e^{t}\right), \Omega(\|x\|)=|x(t)| /(1+|x(t)|)$.

By computation, we have

$$
\begin{gathered}
|h(x)-h(y)| \leq \frac{19}{90}|x-y|, \quad|g(t, x)-g(t, y)| \leq \frac{1}{10}|x-y|, \\
\left|I_{k}(x)-I_{k}(y)\right| \leq \frac{1}{3}|x-y|, \quad|g(t, x)| \leq \frac{1}{10} .
\end{gathered}
$$

Suppose that $x_{0}=h(0)=I_{k}(0)=0$; we have $l_{g}=1 / 10, l_{h}=2 / 9, M=1 / 10, c_{k}=1 / 3$,
$C_{0}=2 M=1 / 5, C=((p-1) /(p q-1))(p-1) / p$
Clearly, for suitable $q \in(0,1)$, all the conditions of Corollary 3.6 are satisfied. So there
exists at least one solution of problem $(4.1)$ on $I$.

\section{Acknowledgments}

The authors would like to thank the Associate Editor and all the anonymous reviewers for their valuable comments and constructive suggestions, which lead to the improvement of the presentation of this paper. This work was funded by Scientific Research Foundation of Nanjing Institute of Technology under Grant Nos. QKJB2010028, QKJA2011009 and also jointly supported by the National Natural Science Foundation of China under Grant Nos. 60874088 and 11072059 and the Specialized Research Fund for the Doctoral Program of Higher Education under Grant No. 20110092110017, the JSPS Innovation Program under Grant CXZZ12_0080.

\section{References}

[1] L. Byszewski, "Theorems about the existence and uniqueness of solutions of a semilinear evolution nonlocal Cauchy problem," Journal of Mathematical Analysis and Applications, vol. 162, no. 2, pp. 494$505,1991$.

[2] L. Byszewski and V. Lakshmikantham, "Theorem about the existence and uniqueness of a solution of a nonlocal abstract Cauchy problem in a Banach space," Applicable Analysis. An International Journal, vol. 40, no. 1, pp. 11-19, 1991.

[3] S. K. Ntouyas and P. Ch. Tsamatos, "Global existence for semilinear evolution equations with nonlocal conditions," Journal of Mathematical Analysis and Applications, vol. 210, no. 2, pp. 679-687, 1997.

[4] M. Benchohra and S. K. Ntouyas, "Nonlocal Cauchy problems for neutral functional differential and integrodifferential inclusions in Banach spaces," Journal of Mathematical Analysis and Applications, vol. 258, no. 2, pp. 573-590, 2001.

[5] J. Liang, J. Liu, and T.-J. Xiao, "Nonlocal Cauchy problems governed by compact operator families," Nonlinear Analysis. Theory, Methods \& Applications, vol. 57, no. 2, pp. 183-189, 2004.

[6] X. Xue, "Existence of solutions for semilinear nonlocal Cauchy problems in Banach spaces," Electronic Journal of Differential Equations, vol. 64, pp. 1-7, 2005.

[7] X. Xue, "Existence of semilinear differential equations with nonlocal initial conditions," Acta Mathematica Sinica, vol. 23, no. 6, pp. 983-988, 2007.

[8] V. Lakshmikantham, D. D. Bainov, and P. S. Simeonov, Theory of Impulsive Differential Equations, World Scientific Publishing, Teaneck, NJ, USA, 1989. 
[9] A. M. Samoilenko and N. A. Perestyuk, Impulsive Differential Equations, World Scientific Publishing, River Edge, NJ, USA, 1995.

[10] N. Abada, M. Benchohra, and H. Hammouche, "Existence and controllability results for impulsive partial functional differential inclusions," Nonlinear Analysis. Theory, Methods E Applications, vol. 69, no. 9, pp. 2892-2909, 2008.

[11] N. Abada, M. Benchohra, and H. Hammouche, "Existence and controllability results for nondensely defined impulsive semilinear functional differential inclusions," Journal of Differential Equations, vol. 246, no. 10, pp. 3834-3863, 2009.

[12] G. M. N'Guérékata, "A Cauchy problem for some fractional abstract differential equation with non local conditions," Nonlinear Analysis. Theory, Methods E Applications, vol. 70, no. 5, pp. 1873-1876, 2009.

[13] G. M. Mophou, "Existence and uniqueness of mild solutions to impulsive fractional differential equations," Nonlinear Analysis. Theory, Methods \& Applications, vol. 72, no. 3-4, pp. 1604-1615, 2010.

[14] F. L. Chen, A. Chen, and X. Wang, "On the solutions for impulsive fractional functional differential equations," Differential Equations and Dynamical Systems, vol. 17, no. 4, pp. 379-391, 2009.

[15] Y. Zhou and F. Jiao, "Existence of mild solutions for fractional neutral evolution equations," Computers E Mathematics with Applications. An International Journal, vol. 59, no. 3, pp. 1063-1077, 2010.

[16] Y. Zhou and F. Jiao, "Nonlocal Cauchy problem for fractional evolution equations," Nonlinear Analysis. Real World Applications. An International Multidisciplinary Journal, vol. 11, no. 5, pp. 4465-4475, 2010.

[17] M. Benchohra, J. Henderson, S. K. Ntouyas, and A. Ouahab, "Existence results for fractional functional differential inclusions with infinite delay and applications to control theory," Fractional Calculus \& Applied Analysis. An International Journal for Theory and Applications, vol. 11, no. 1, pp. 35$56,2008$.

[18] M. Benchohra and B. A. Slimani, "Existence and uniqueness of solutions to impulsive fractional differential equations," Electronic Journal of Differential Equations, vol. 120, pp. 1-11, 2009.

[19] A. P. Chen and Y. Chen, "Existence of solutions to anti-periodic boundary value problem for nonlinear fractional differential equations with impulses," Advances in Difference Equations, Article ID 915689, 17 pages, 2011.

[20] S. Hamani, M. Benchohra, and J. R. Graef, "Existence results for boundary-value problems with nonlinear fractional differential inclusions and integral conditions," Electronic Journal of Differential Equations, p. No. 20, 16, 2010.

[21] Y.-K. Chang and J. J. Nieto, "Some new existence results for fractional differential inclusions with boundary conditions," Mathematical and Computer Modelling, vol. 49, no. 3-4, pp. 605-609, 2009.

[22] B. Ahmad, J. J. Nieto, and J. Pimentel, "Some boundary value problems of fractional differential equations and inclusions," Computers E Mathematics with Applications. An International Journal, vol. 62, no. 3, pp. 1238-1250, 2011.

[23] B. Ahmad and S. Sivasundaram, "Existence results for nonlinear impulsive hybrid boundary value problems involving fractional differential equations," Nonlinear Analysis. Hybrid Systems. An International Multidisciplinary Journal, vol. 3, no. 3, pp. 251-258, 2009.

[24] K. Balachandran and S. Kiruthika, "Existence of solutions of abstract fractional impulsive semilinear evolution equations," Electronic Journal of Qualitative Theory of Differential Equations, p. No. 4, 12, 2010.

[25] K. Balachandran, S. Kiruthika, and J. J. Trujillo, "Existence results for fractional impulsive integrodifferential equations in Banach spaces," Communications in Nonlinear Science and Numerical Simulation, vol. 16, no. 4, pp. 1970-1977, 2011.

[26] R. P. Agarwal, M. Benchohra, and S. Hamani, "A survey on existence results for boundary value problems of nonlinear fractional differential equations and inclusions," Acta Applicandae Mathematicae. An International Survey Journal on Applying Mathematics and Mathematical Applications, vol. 109, no. 3, pp. 973-1033, 2010.

[27] R. P. Agarwal and B. Ahmad, "Existence theory for anti-periodic boundary value problems of fractional differential equations and inclusions," Computers $\mathcal{E}$ Mathematics with Applications. An International Journal, vol. 62, no. 3, pp. 1200-1214, 2011.

[28] A. P. Chen and Y. Chen, "Existence of solutions to anti-periodic boundary value problem for nonlinear fractional differential equations," Differential Equations and Dynamical Systems, vol. 19, no. 3, pp. 237252, 2011.

[29] B. C. Dhage, A. Boucherif, and S. K. Ntouyas, "On periodic boundary value problems of first-order perturbed impulsive differential inclusions," Electronic Journal of Differential Equations, vol. 84, pp. 1-9, 2004.

[30] A. Lasota and Z. Opial, "An application of the Kakutani-Ky-Fan theorem in the theory of ordinary differential equations," Bulletin de l'Académie Polonaise des Sciencess, vol. 13, pp. 781-786, 1965. 
[31] J.-P. Aubin and A. Cellina, Differential Inclusions, Springer-Verlag, Berlin, Germany, 1984.

[32] K. Deimling, Multivalued Differential Equations, Walter de Gruyter, Berlin, Germany, 1992.

[33] I. Podlubny, Fractional Differential Equations, Academic Press, San Diego, Calif, USA, 1999.

[34] S. Zhang, "Positive solutions for boundary-value problems of nonlinear fractional differential equations," Electronic Journal of Differential Equations, vol. 36, pp. 1-12, 2006. 


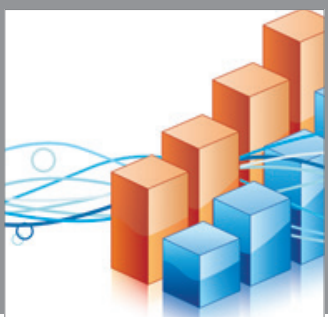

Advances in

Operations Research

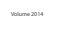

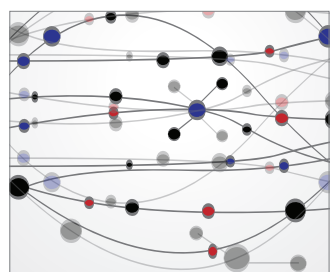

\section{The Scientific} World Journal
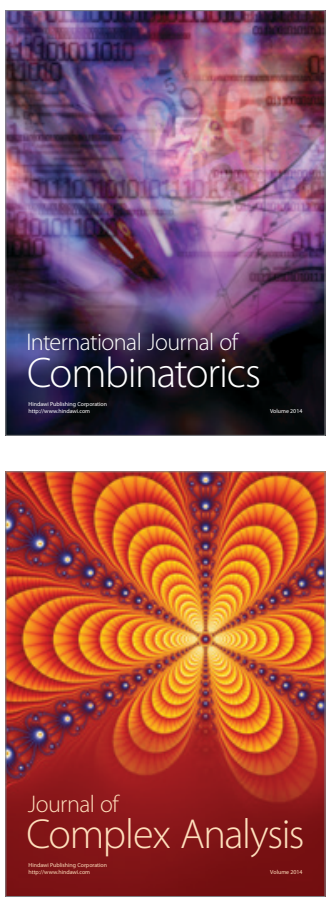

International Journal of

Mathematics and

Mathematical

Sciences
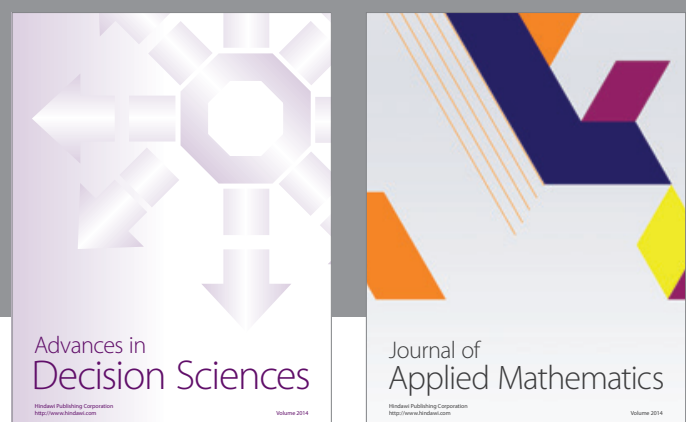

Journal of

Applied Mathematics
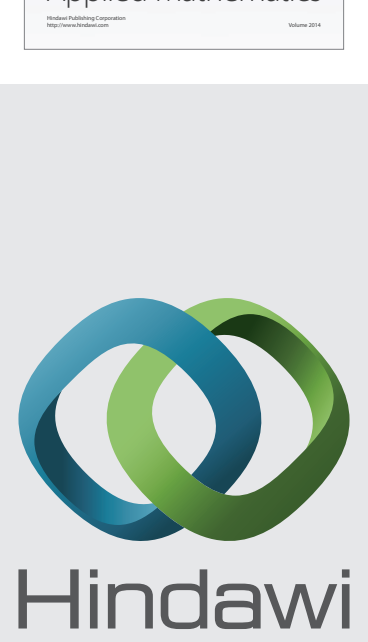

Submit your manuscripts at http://www.hindawi.com
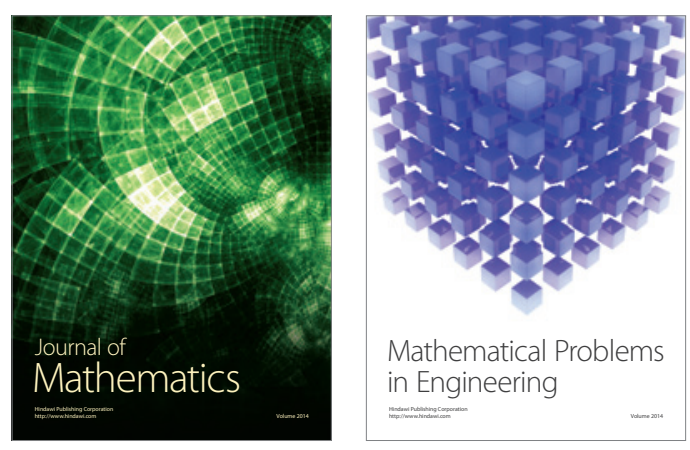

Mathematical Problems in Engineering
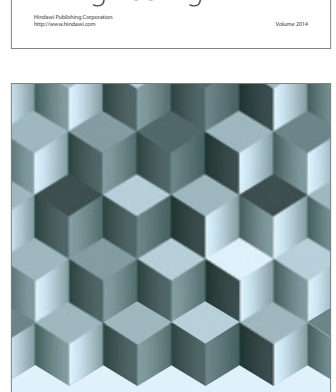

Journal of

Function Spaces
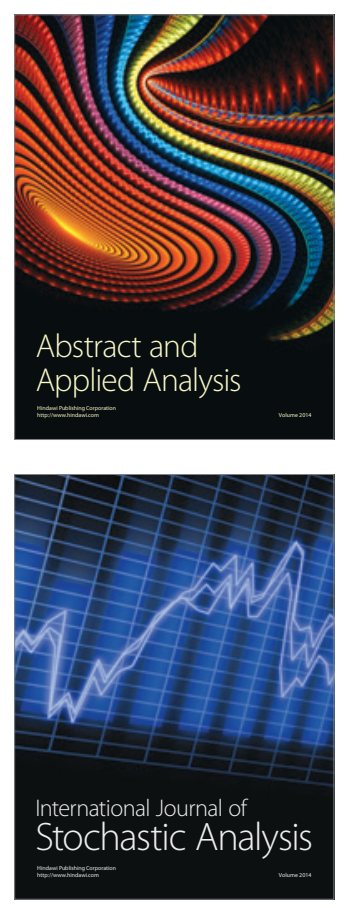

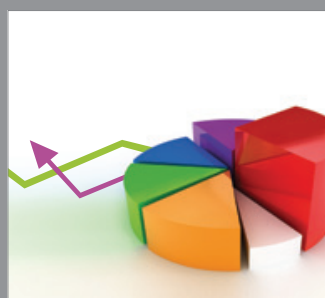

ournal of

Probability and Statistics

Promensencen
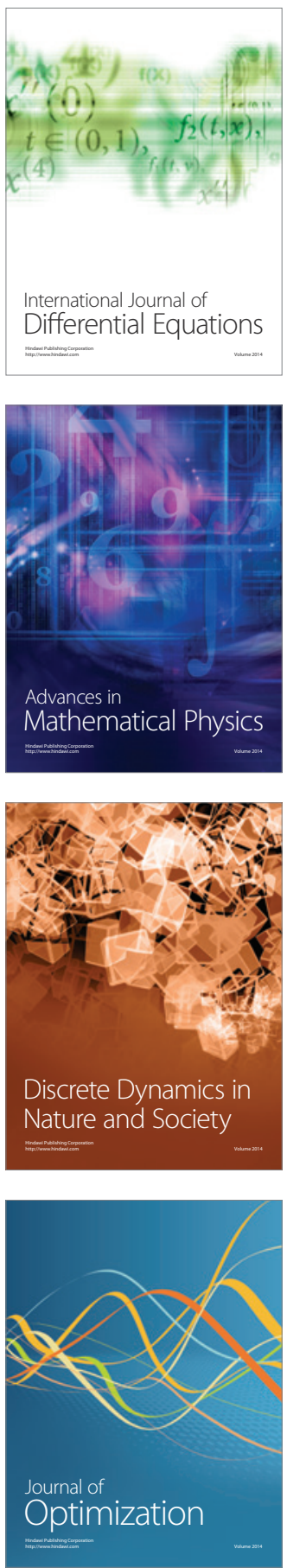